\title{
Leucine 7 to proline 7 polymorphism in the neuropeptide $Y$ gene and changes in serum lipids during a family-based counselling intervention among school-aged children with a family history of CVD
}

\author{
Marika Salminen ${ }^{1, *}$,Terho Lehtimäki ${ }^{2}$, Yue-Mei Fan ${ }^{2}$, Tero Vahlberg ${ }^{3}$ and \\ Sirkka-Liisa Kivelä ${ }^{1,4,5}$ \\ ${ }^{1}$ Institute of Clinical Medicine, Family Medicine, University of Turku, Lemminkäisenkatu 1, Fl-20014 University of \\ Turku, Finland: ${ }^{2}$ Laboratory of Atherosclerosis Genetics, Department of Clinical Chemistry, Centre for Laboratory \\ Medicine, Tampere University Hospital and University of Tampere Medical School, Tampere, Finland: ${ }^{3}$ Institute of \\ Clinical Medicine, Biostatistics, University of Turku, Turku, Finland: ${ }^{4}$ Satakunta Central Hospital, Pori, Finland: \\ ${ }^{5}$ Unit of Family Medicine, Turku University Hospital, Turku, Finland
}

Submitted 17 April 2007: Accepted 7 December 2007: First published online 18 February 2008

\begin{abstract}
Objective: To compare whether serum lipids and their changes during a health education intervention are associated with the Leu7Pro polymorphism in the signal peptide part of neuropeptide Y (NPY) in children with normal weight and in those with overweight.

Design: An intervention study.

Setting: A family-based intervention of risk factors for prevention of CHD in Finland.

Subjects: Subjects were 443 children with a family history of CVD participating in family-based health education. The children were divided into two groups according to NPY genotype: children with Leu7/Pro7 or Pro7/Pro7 genotype ( $n$ 50) and children with Leu7/Leu7 genotype ( $n$ 393). The final sample of the follow-up study included 353 (80\%) children (Pro7 allelle carriers, $n$ 43; Leu7/Leu7, $n$ 310).

Results: At baseline, the Leu7Pro polymorphism was not associated with serum lipid values after adjustment for body weight in boys or girls. There was a significant interaction of NPY genotype group by time and body weight $(P=0 \cdot 043$ for three-way interaction: time $\times$ NPY genotype $\times$ body weight) in LDL-cholesterol (LDL-C) concentration among boys. LDL-C decreased among boys with normal weight in both NPY groups and in overweight boys with the Leu7/Leu7 genotype, whereas it increased in overweight boys with the Pro7 allele. Two-way interaction (time $\times$ NPY genotype) showed no significant differences in changes of serum lipids between the NPY genotype groups among boys or girls.

Conclusions: The Leu7Pro polymorphism may be associated with dietary response to LDL-C concentration in overweight boys with a family history of early-onset CVD.
\end{abstract}

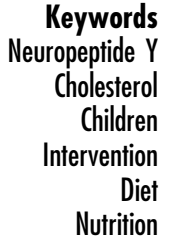

Neuropeptide Y (NPY) is a neurotransmitter that is widely expressed in the central ${ }^{(1)}$ and peripheral $^{(2)}$ nervous system. The Pro7 allele in the gene encoding NPY has been associated with elevated serum total cholesterol (TC) $^{(3-5)}$ and LDL-cholesterol (LDL-C) $)^{(3,4,6)}$ concentrations and with $\mathrm{TAG}^{(7)}$ among adults. The association of the Pro7 allele with elevated serum cholesterol concentrations is stronger in obese subjects ${ }^{(3,4)}$.

Few studies about the association between NPY polymorphism and serum lipid concentrations have been carried out in children. The Leu7Pro polymorphism was associated with serum TAG values in boys at ages 5, 7 and 9 years, but not with TC or LDL-C values among either boys or girls ${ }^{(8,9)}$. Dietary intervention did not affect the association of Leu7Pro polymorphism with serum lipids. The results of a study about the impact of Leu7Pro polymorphism on the response of serum lipids to a reduced-fat diet showed no difference between the NPY genotype groups among slightly obese adults ${ }^{(10)}$.

The present study was undertaken to determine whether the Leu7Pro polymorphism in NPY modifies serum lipid values and their changes during a family-based health 
education of school-aged children with a family history of CVD. As the association of the Pro7 allele with elevated serum cholesterol concentrations seems to be stronger in obese subjects, we included body weight in the statistical models.

\section{Methods}

\section{Subjects}

The intervention group of children and adolescents for the family-based health education/counselling was selected from a population aged 6 to 17 years living in Kainuu (ten municipalities in eastern Finland) and having a family history of early-onset (first attack before the age of 55 years among men and 65 among women) CHD, myocardial infarction (MI) or brain infarction (BI) in their parents or grandparents, or having a history of familial hypercholesterolaemia (FH) (high-risk families). The parents, sisters and brothers of children in the intervention group also participated in the health counselling.

The names of adult residents in Kainuu having had early-onset MI, CHD or BI, or a history of $\mathrm{FH}$, during 1987-1995 were collected from hospital discharge registers (International Classification of Diseases version 9, codes: 272, 410-414) ${ }^{(11)}$ (Fig. 1). They were informed by letter about the project and asked to report the names and addresses of their children and grandchildren aged 6-17 years living in Kainuu. The names and addresses of 600 children were received.

Basic examinations were carried out from September 1997 to May 1998. Participation was voluntary, and written informed consent was given by the children themselves ( 15 years or above) or by their parents (for children under 15 years). The Ethical Committee of Oulu University approved the plan.

\section{Neuropeptide Y genotype analysis}

DNA was extracted from peripheral blood leucocytes using a commercially available kit (Qiagen Inc., Valencia, CA, USA). NPY genotypes were determined by PCR and restriction enzyme digestion ${ }^{(3)}$. The primers used were 5'-CCCGTCCGTTGAGCCTTCTG-3' and 5'-CGGTCCCGCG GTCCC-3'. The amplification cycle was performed in a PTC225 thermal cycler (MJ Research Inc., Watertown, MA, USA). After initial denaturation at $94^{\circ} \mathrm{C}$ for $5 \mathrm{~min}$, the DNA was amplified by 30 cycles of $1 \mathrm{~min}$ at $94^{\circ} \mathrm{C}, 1 \mathrm{~min}$ at $69^{\circ} \mathrm{C}$ and $1 \mathrm{~min}$ at $72^{\circ} \mathrm{C}$, with a final elongation step of $10 \mathrm{~min}$ at $72^{\circ} \mathrm{C}$. The PCR products were digested by BsiEI (New England Biolabs, Ipswich, MA, USA) and the digested fragments separated by electrophoresis on $2 \%$ agarose gel. The PCR products were cut into fragments 48, 190 and $238 \mathrm{bp}$ long, respectively. The PCR product not containing a BsiEI restriction enzyme site yields a $238 \mathrm{bp}$ band; the heterozygotes yield bands of 238, 190 and 48bp; and the Pro7/ Pro7 homozygotes give bands of 190 and $48 \mathrm{bp}$.

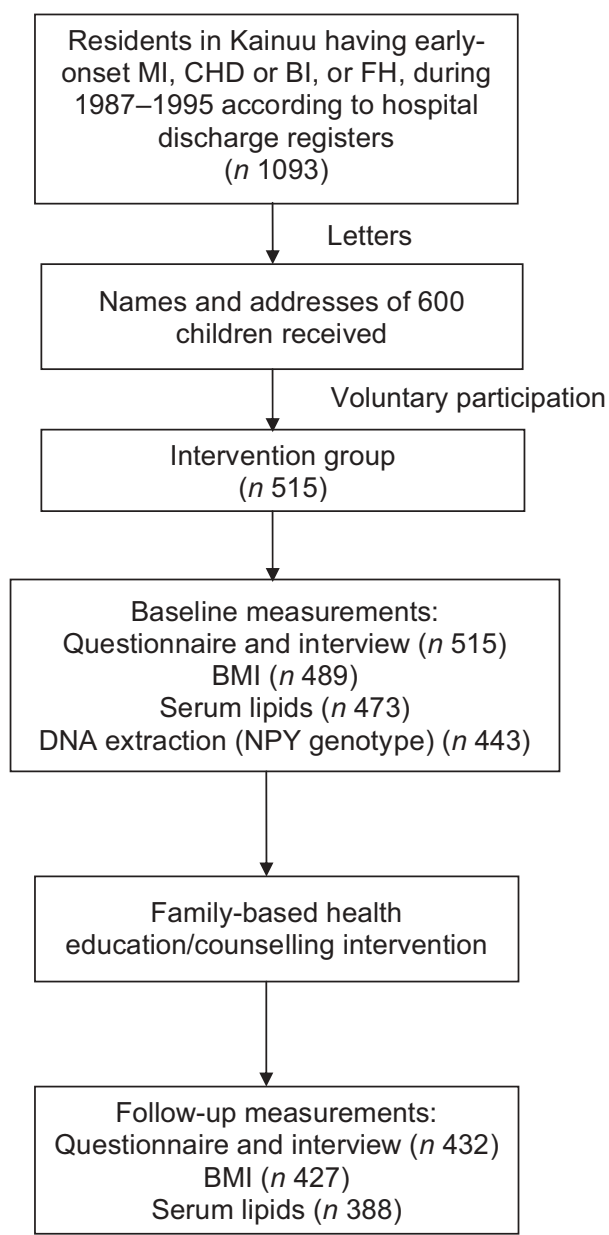

1997-98

Fig. 1 Flow chart of the intervention (MI, myocardial infarction; $\mathrm{BI}$, brain infarction; $\mathrm{FH}$, familial hypercholesterolaemia; NPY, neuropeptide $Y$ )

The NPY genotype of 443 children was determined. The children were divided into two groups according to their NPY genotype: children with the Pro7 allele (Leu7/Pro7 or Pro7/Pro7) ( $n 50$; forty-eight heterozygotes and two homozygotes, twenty-two boys and twenty-eight girls) and children with the Leu7/Leu7 genotype ( $n$ 393; 202 boys and 191 girls). The final sample of the follow-up study included $353(80 \%)$ children (forty-three and 310 children in each genotype group, respectively) (Fig. 2). The genotype analyses were carried out at the Department of Clinical Chemistry at the University of Tampere and Tampere University Hospital (Laboratory of Atherosclerosis Genetics).

\section{Lipid analyses}

Blood samples were drawn in the laboratories of the ten health centres in Kainuu after a period of fasting of at least $10 \mathrm{~h}$. The sera were separated and the samples were sent to the laboratory of Kainuu Central Hospital, where they were kept frozen at $-20^{\circ} \mathrm{C}$ until analysed. Serum TC concentration was analysed using the cholesterol esterase 


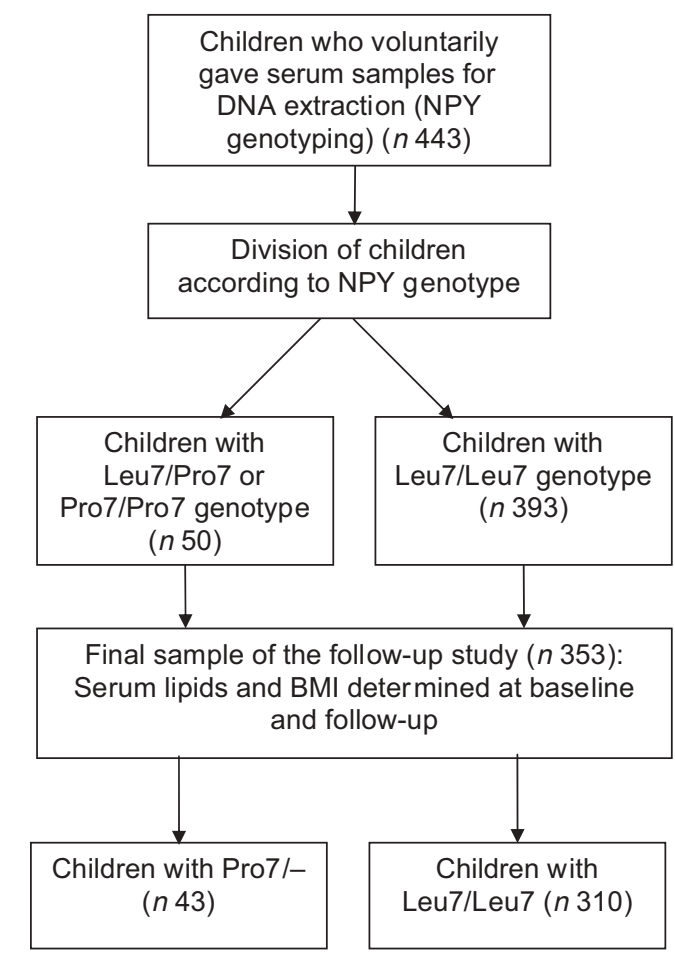

Fig. 2 Flow chart of the study material (NPY, neuropeptide Y)

method (Konelab, Espoo, Finland) ${ }^{(12-15)}$. Very-low-density lipoprotein and LDL were precipitated with a phosphowolframate-magnesium chloride reagent and separated by centrifugation. After this, HDL-cholesterol (HDL-C) was determined enzymatically. TAG were analysed using the glycerol phosphorylase method (Konelab, Espoo, Finland). LDL-C was calculated using Friedewald's formula ${ }^{(16)}$. Concentrations were expressed as $\mathrm{mmol} / \mathrm{l}$.

\section{Assessment of normal weight and overweight}

The height and weight of the participants were measured by two public health nurses and BMI was calculated. Classification of normal weight and overweight or obesity was determined according to BMI using internationally acceptable age- and sex-specific cut-off points ${ }^{(17)}$.

\section{Other measurements}

Two trained public health nurses took measurements of blood pressure and triceps skinfold thickness. With the help of the parents, all participating children filled out structured questionnaires pertaining to diet and nutrition, exercise and other health issues. Answers were complemented with interviews carried out by the public health nurses of Kainuu Heart Association, and the interview included questions on smoking and the use of alcohol and drugs.

\section{Family-based bealtb education/counselling intervention}

All children participated in a family-based health education/counselling intervention throughout the study.
Health education/counselling began in September 1997 and the follow-up measurements were completed in the spring of 2000. The intervention consisted of two individual counselling sessions with children at schools (1 h) and three counselling sessions with children and their families in their homes (2-3h). Two trained public health nurses worked as counsellors with each having their own designated children and families. Children and other family members were individually counselled about diet and nutrition (fatty acids, fibre content, salt), overweight, exercise, cigarette smoking and drugs and alcohol during the family sessions. Diet and nutrition were stressed in the intervention. The intervention has been described in detail elsewhere ${ }^{(18)}$.

Among children with starting concentrations of TC between 5.0 and $6.5 \mathrm{mmol} / \mathrm{l}$, TC concentrations were measured at health centres at a 6-month interval for follow-up purposes. Children whose serum TC concentration was $6.5 \mathrm{mmol} / \mathrm{l}$ or more at the beginning ( $n$ 20) were treated in Kainuu Central Hospital. In addition to participating in the family-based health education/counselling ( $n$ 16), the children were counselled and treated by regular health services. No lipid-lowering drugs were used.

Follow-up measurements similar to those in the basic examination except for determining NPY genotype were carried out from January 2000 to June 2000. Of the 443 original participants, ninety dropped out during the follow-up period (seven with the Pro7 substitution and eighty-three with the Leu7/Leu7 genotype) because of relocation, parents' divorce, family member's serious illness or other reasons. Only children with NPY genotype determined at baseline and with complete baseline and follow-up data about lipids, as well as BMI, were included in the analyses ( $n$ 353) (80\%). Of the 353 children and adolescents included in the analyses, forty-three (nineteen boys and twenty-four girls) had the Pro7 substitution and 310 (157 boys and 153 girls) had the Leu7/Leu7 genotype.

\section{Statistical analyses}

At baseline, the mean ages between the NPY genotype groups were compared with the two-sample $t$ test. Fisher's exact test was used to analyse the differences between the NPY genotype groups at baseline in the proportions of participants with normal weight and with overweight or obesity and in the proportions of smoking and non-smoking children. The differences in serum lipids between the NPY genotype groups were evaluated with ANOVA where body weight was used as a covariate.

The differences in changes in serum lipids between the NPY genotype groups (two-way interaction: time $\times$ NPY genotype) and between the NPY genotype and body weight groups (three-way interaction: time $\times$ NPY genotype $\times$ body weight) were tested using ANOVA for repeated measurements. Due to a skewed distribution, TAG concentrations were log-transformed for statistical 
Table 1 Baseline characteristics of the study population by neuropeptide $\mathrm{Y}$ genotype group and gender

\begin{tabular}{|c|c|c|c|c|c|c|c|c|c|c|}
\hline & \multicolumn{5}{|c|}{ Boys } & \multicolumn{5}{|c|}{ Girls } \\
\hline & \multicolumn{2}{|c|}{ Pro7/- (n 19) } & \multicolumn{2}{|c|}{ Leu7/Leu7 (n 157) } & \multirow[b]{2}{*}{$P$} & \multicolumn{2}{|c|}{ Pro7/- (n 24) } & \multicolumn{2}{|c|}{ Leu7/Leu7 ( $n$ 153) } & \multirow[b]{2}{*}{$P$} \\
\hline & Mean & SD & Mean & SD & & Mean & SD & Mean & SD & \\
\hline Age (years) & $11 \cdot 0$ & $3 \cdot 1$ & $10 \cdot 9$ & $2 \cdot 9$ & $0 \cdot 857$ & $10 \cdot 9$ & $2 \cdot 9$ & $10 \cdot 8$ & $2 \cdot 9$ & 0.961 \\
\hline $\mathrm{TC}(\mathrm{mmol} / \mathrm{l})$ & $4 \cdot 6$ & 0.9 & $4 \cdot 6$ & $0 \cdot 8$ & 0.876 & $4 \cdot 8$ & 0.8 & $4 \cdot 8$ & 0.9 & 0.984 \\
\hline LDL-C (mmol/l) & $2 \cdot 7$ & $0 \cdot 8$ & $2 \cdot 7$ & $0 \cdot 6$ & 0.942 & $2 \cdot 9$ & 0.7 & $2 \cdot 9$ & 0.9 & 0.769 \\
\hline HDL-C (mmol/li) & 1.5 & $0 \cdot 3$ & $1 \cdot 5$ & $0 \cdot 4$ & 0.992 & 1.5 & $0 \cdot 3$ & $1 \cdot 6$ & $0 \cdot 3$ & 0.235 \\
\hline \multirow[t]{2}{*}{ TAG $(\mathrm{mmol} / \mathrm{l})$} & 0.8 & 0.4 & 0.8 & $0 \cdot 4$ & 0.541 & 0.9 & 0.4 & 0.8 & $0 \cdot 3$ & 0.395 \\
\hline & $n$ & $\%$ & $n$ & $\%$ & & $n$ & $\%$ & $n$ & $\%$ & \\
\hline \multicolumn{11}{|l|}{ Current smoking } \\
\hline No & 15 & 79 & 137 & 87 & & 17 & 71 & 124 & 81 & \\
\hline Yes & 4 & 21 & 20 & 13 & 0.300 & 7 & 29 & 29 & 19 & 0.277 \\
\hline \multicolumn{11}{|l|}{ Body weight* } \\
\hline Normal weight & 14 & 74 & 115 & 73 & & 21 & 88 & 125 & 82 & \\
\hline Overweight or obese & 5 & 26 & 42 & 27 & $1 \cdot 000$ & 3 & 13 & 28 & 18 & 0.772 \\
\hline
\end{tabular}

TC, total cholesterol; LDL-C, LDL-cholesterol; HDL-C, HDL-cholesterol.

${ }^{*}$ Classification of normal weight and overweight/obesity was determined according to BMI using internationally acceptable age- and sex-specific cut-off points ${ }^{(17)}$.

analyses. All analyses were done separately for boys and girls. $P$ values of less than 0.05 were considered statistically significant. Data are expressed as means with their standard deviation unless otherwise stated. Statistical analyses were carried out using the SAS System for Windows statistical software package release 8.02 (SAS Institute, Cary, NC, USA).

\section{Results}

\section{Baseline characteristics}

There were no significant differences in baseline characteristics between the NPY genotype groups among either boys or girls (Table 1 ).

\section{Neuropeptide Y Leu 7Pro polymorphism and baseline serum lipids}

At baseline, there were no significant differences in serum lipid values between the NPY genotype groups adjusted by body weight among either boys or girls.

\section{Neuropeptide Y Leu 7Pro polymorphism and changes in serum lipids during the intervention}

The only significant interaction of the NPY genotype groups by time and body weight was found in LDL-C concentration among boys (three-way interaction: time $\times$ NPY genotype $\times$ body weight, $P=0 \cdot 043$ ). LDL-C level decreased among boys with normal weight in both NPY genotype groups, whereas among boys who were overweight it decreased in those with the Leu7/Leu7 genotype and increased in those with Pro7 substitution (Fig. 3).

There were no significant interactions of the NPY groups by time (two-way interaction: time $\times$ NPY genotype) with

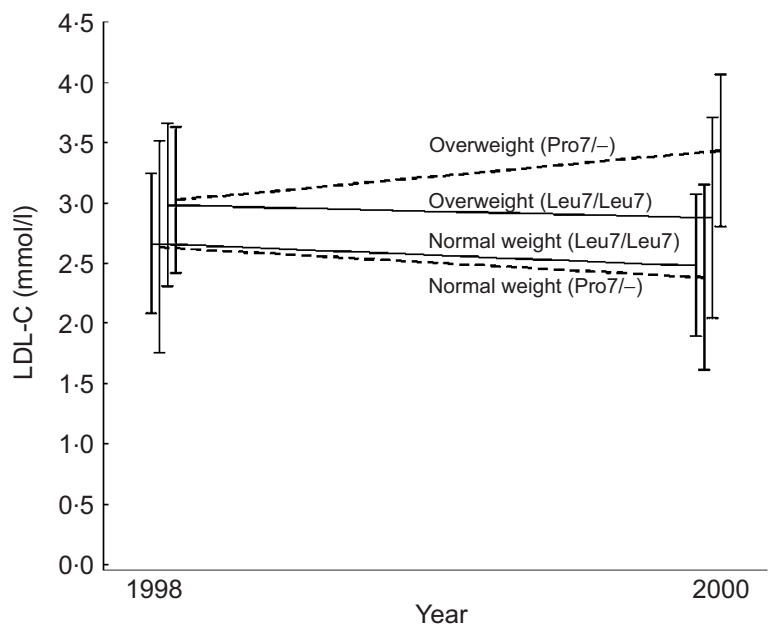

Fig. 3 Changes of LDL-cholesterol (LDL-C) concentration $(\mathrm{mmol} / \mathrm{l})$ by neuropeptide $\mathrm{Y}$ (NPY) genotype and body weight (three-way interaction: time $\times$ NPY genotype $\times$ body weight, $P=0.043$ ) among boys (overweight boys with Pro7 substitution, $n 5$; overweight boys with Leu7/Leu7 genotype, $n$ 42; normal-weight boys with Pro7 substitution, $n$ 115; normal-weight boys with Leu7/Leu7 genotype, $n$ 14). Values are means with their standard deviation represented by vertical bars

any of the serum lipids among boys or girls, indicating that there were no significant differences in changes between the groups during the intervention.

Between subjects with normal weight and those who were overweight, after adjustment for NPY genotype, there were significant differences only in changes of $\mathrm{TC}(-0.3(\mathrm{SD} 0.5) v .-0 \cdot 1(\mathrm{SD} 0 \cdot 9) \mathrm{mmol} / \mathrm{l} ; P=0 \cdot 040)$ and LDL-C $(-0 \cdot 2(\operatorname{sD~} 0 \cdot 4) v .-0 \cdot 1(\mathrm{SD} 0 \cdot 7) \mathrm{mmol} / \mathrm{l} ; P=0 \cdot 012)$ concentrations among boys (two-way interaction: time $X$ body weight). 


\section{Discussion}

The overall goal of the present family-based education intervention was to modify or change undesirable health habits of all family members, reduce high TC and LDL-C levels and lower overall risk for CHD, MI, BI or FH among the members of those families. The intervention used the health education approach within the family and concentrated specifically on counselling children and adolescents about nutrition, exercise and cigarette smoking. The intervention had favourable effects on the children's use of fats $^{(18)}$. The effects of the intervention on serum cholesterol and blood pressure values were also reported earlier for the same population of children ${ }^{(19)}$. This previous study, with an appropriate control group but without analyses about genotypes, indicated that family-oriented health counselling had favourable effects on TC and LDL-C concentrations among girls aged 6-9 years, and on the development of diastolic blood pressure among boys aged 6-9 years, compared with corresponding controls not participating in such an intervention. We do not know the actual saturated fat and cholesterol contents of diets at baseline and follow-up because the nutritional data were collected with questionnaires instead of diaries. As a consequence, the results do not allow any conclusions to be drawn on the relative contributions of the reductions in intake amounts of these components.

The primary aim of the present study was to compare the effects of the intervention on serum lipids between subjects with different NPY Leu7Pro genotypes in the intervention group. Moreover, since earlier studies showed stronger associations of NPY Leu7Pro genotypes with serum cholesterol levels in obese subjects, we also took body weight into account in the statistical analyses. The data of the present study comprised those of children with their NPY genotype determined at baseline and with complete baseline and follow-up data about serum lipids and BMI. Because the changes in lipid profiles of boys and girls were partly different, results are presented separately for boys and girls.

At baseline of the study, there were no significant differences in serum lipid values between the NPY genotype groups after adjustment for body weight. One earlier study conducted among pre-school children showed that the Pro7 substitution was associated only with 14-17\% higher mean TAG in boys as compared with the Leu7/Leu7 genotype ${ }^{(8)}$. Studies carried out among adults have shown that the presence of Pro7 in NPY is associated with higher TAG and TC levels of type 1 diabetic patients ${ }^{(7)}$, higher TC level of CHD patients ${ }^{(5)}$ and higher TC and LDL-C ${ }^{(3,4)}$ concentrations among obese and normal-weight subjects ${ }^{(3)}$.

The only significant interaction of the NPY genotype groups by time and body weight (three-way interaction) during the follow-up period was found in LDL-C concentration among boys. LDL-C decreased among normal-weight boys in both NPY genotype groups and in overweight boys without the Pro7/- genotype, whereas it increased in overweight boys with the Pro7/- genotype. There were only five overweight boys with the Pro7 substitution, which has to be taken into account in the interpretation of the results. No significant interactions of the NPY genotype groups by time (two-way interaction) were found with any of the serum lipids. An earlier study among slightly obese middle-aged subjects showed no difference in the response to a reduced-fat diet between NPY genotype groups ${ }^{(10)}$. Previous publications have reported that the Pro7 substitution in NPY is associated with higher serum TAG levels in boys ${ }^{(8,9)}$. Pro7 substitution might have an effect on the composition of lipid particles mediated by the lipoprotein and hepatic lipase activity that affects lipid metabolism ${ }^{(10)}$. The reason why this effect is observed only in boys may be related to gender differences in the body fat proportion in childhood (girls having approximately 50\% more body fat than boys) ${ }^{(20)}$.

The education and counselling in our study was not very intensive. During 33 months there were two counselling sessions at the children's schools and three at their homes, amounting to about $8-11 \mathrm{~h}$ of individual counselling per child and/or family. However, the intervention had favourable effects on the use of fats and serum lipid levels of children. There are only a few previous studies about the associations of NPY gene variation with plasma lipid responses to reduced dietary fat. It cannot be concluded whether a more intensive intervention would give different results regarding the effect of NPY genotype on plasma lipid responses. However, the plasma TC and LDL-C levels in children aged 4-11 years with less family history of CVD have been found to be significantly more responsive to a change in dietary cholesterol than levels in children with a stronger family history of $\mathrm{CVD}^{(21)}$.

The exact mechanism by which Leu7Pro genotype may affect the effects of a dietary intervention is not known. The Pro7 substitution may have an influence on NPY processing, circulating NPY levels, growth hormone secretion, serum cholesterol levels ${ }^{(3,22-24)}$ and blood pressure. The Leu7Pro polymorphism is associated with high birth weight and high serum TAG in young children, and with enhanced atherosclerosis in elderly subjects. Thus, the effects may be linked to a better compliance or other aspects of behaviour, to a different sensitivity of lipid levels to dietary and other manipulations in different genotypes, or even to a mixture of these different mechanisms. We are not able to provide any new direct mechanistic explanation by our family-based intervention study conducted with children.

The results of the present study may have been influenced by the large variation of children's age (6-17 years). There are age- and gender-related changes in serum lipid levels of children. Serum cholesterol concentration decreases during puberty. This is due to the decline in LDL-C level among boys and girls and to the decline in HDL-C level among boys ${ }^{(25)}$. 
According to our major findings the observed effects on LDL-C are exclusively found in overweight boys but not in boys with normal weight. It may be that, in patients with serum lipid disorders or in overweight patients participating in more intensive intervention regimes, the Leu7Pro polymorphism may have a stronger association with altered serum lipid levels. This point requires further investigation with controlled experimental studies.

\section{Acknowledgements}

No conflict of interest.

Authors' contributions: S.-L.K. planned and organised the family-based counselling intervention trial. M.S. conceptualised the design of this study together with T.L. and S.-L.K. NPY genotype analysis was done by Y.-M.F. Statistical analyses and interpretation of the data were made by M.S., T.V. and S.-L.K. Writing and critical revision of the manuscript were accomplished by all authors. Funding was obtained by S.-L.K. together with T.L.

Sources of funding: The authors wish to acknowledge Finland's Slot Machine Association, the Ministry of Social Affairs and Health, the Juho Vainio Foundation, the Yrjö Jahnsson Foundation, the Medical Research Fund of Tampere University Hospital, the Finnish Foundation for Cardiovascular Research, the Academy of Finland (grant no. 104821), the Emil Aaltonen Foundation (T.L.), the Elli and Elvi Oksanen Fund of the Pirkanmaa Fund under the auspices of the Finnish Cultural Foundation, The Pirkanmaa Regional Fund of the Finnish Cultural Foundation, the Research Foundation of Orion Corporation and the Ida Montin Foundation for financial support for the study.

\section{References}

1. Gray TS \& Morley JE (1986) Neuropeptide Y: anatomical distribution and possible function in mammalian nervous system. Life Sci 38, 389-401.

2. Lundberg JM, Terenius L, Hokfelt T, Martling CR, Tatemoto K, Mutt V, Polak J, Bloom S \& Goldstein M (1982) Neuropeptide Y (NPY)-like immunoreactivity in peripheral noradrenergic neurons and effects of NPY on sympathetic function. Acta Physiol Scand 116, 477-480.

3. Karvonen MK, Pesonen U, Koulu M, Niskanen L, Laakso M, Rissanen A, Dekker JM, Hart LM, Valve R \& Uusitupa MI (1998) Association of a leucine(7)-to-proline(7) polymorphism in the signal peptide of neuropeptide $\mathrm{Y}$ with high serum cholesterol and LDL cholesterol level. Nat Med 4, 1434-1437.

4. Karvonen MK, Valkonen V-P, Lakka TA et al. (2001) Leucine7 to proline7 polymorphism in the preproneuropeptide $\mathrm{Y}$ is associated with the progression of carotic atherosclerosis, blood pressure and serum lipids in Finnish men. Atherosclerosis 159, 145-151.

5. Erkkilä AT, Lindi V, Lehto S, Laakso M \& Uusitupa MIJ (2002) Association of leucine 7 to proline 7 polymorphism in the preproneuropeptide $\mathrm{Y}$ with serum lipids in patients with coronary heart disease. Mol Genet Metab 75, 260-264.
6. Nyström F, Nilsson P, Olsson AG, Karlberg BE \& Ohman KP (1996) A population study of plasma neuropeptide Y: correlations with components of the metabolic syndrome. Blood Press 5, 349-353.

7. Pettersson-Fernholm K, Karvonen MK, Kallio J, Forsblom CM, Koulu M, Pesonen U, Fagerudd JA, Groop PH; FinnDiane Study Group (2004) Leucine 7 to proline 7 polymorphism in the preproneuropeptide $\mathrm{Y}$ is associated with proteinuria, coronary heart disease, and glycemic control in type 1 diabetic patients. Diabetes Care 27, 503-509.

8. Karvonen MK, Koulu M, Pesonen U, Uusitupa MI, Tammi A, Viikari J, Simell O \& Rönnemaa T (2000) Leucine 7 to proline 7 polymorphism in the preproneuropeptide $\mathrm{Y}$ is associated with birth weight and serum triglyceride concentration in preschool-aged children. J Clin Endocrinol Metab 85, 1455-1460.

9. Karvonen MK, Ruottinen S, Koulu M, Pesonen U, Niinikoski H, Rask-Nissilä L, Simell O \& Rönnemaa T (2006) Nutrient intake, weight, and Leu7Pro polymorphism in prepro-neuropeptide $\mathrm{Y}$ in children. J Clin Endocrinol Metab 91, 4664-4668.

10. Schwab US, Ågren JJ, Valve R et al. (2002) The impact of the leucine 7 to proline 7 polymorphism of the neuropeptide $\mathrm{Y}$ gene on postprandial lipemia and on the response of serum total and lipoprotein lipids to a reduced fat diet. Eur J Clin Nutr 56, 149-156.

11. World Health Organization (1997) International Classification of Diseases, 1975 Revision. Manual of the International Statistical Classification of Diseases, Injuries, and Causes of Death, vol. 1. Geneva: WHO.

12. Lopes-Virella MF, Stone P, Ellis S \& Colwell JA (1977) Cholesterol determination in high-density lipoproteins separated by three different methods. Clin Chem 23, 882-884.

13. Fossati P \& Prencipe L (1982) Serum triglycerides determined colorimetrically with an enzyme that produces hydrogen peroxide. Clin Chem 28, 2077-2080.

14. Assmann G, Schriewer H, Schmitz G \& Hagele EO (1983) Quantification of high-density-lipoprotein cholesterol by precipitation with phosphotungstic acid $/ \mathrm{MgCl}_{2}$. Clin Chem 29, 2026-2030.

15. Tietz NW (1990) Clinical Guide to Laboratory Test. Philadelphia, PA: WB Saunders Company.

16. Friedewald WT, Levy R \& Fredrickson DS (1972) Estimation of the concentration of low-density lipoprotein cholesterol in plasma without use of the preparative ultracentrifuge. Clin Chem 18, 499-502.

17. Cole TJ, Bellizzi MC, Flegal KM \& Dietz WH (2000) Establishing a standard definition for child overweight and obesity worldwide: international survey. BMJ 320, 1240-1246.

18. Salminen M, Vahlberg T, Ojanlatva A \& Kivelä S-L (2005) Effects of a controlled family-based health education/ counseling intervention. Am J Health Behav 29, 395-406.

19. Salminen M, Vahlberg T \& Kivelä S-L (2005) Effects of family-oriented risk-based prevention on serum cholesterol and blood pressure values of children and adolescents. Scand J Prim Health Care 23, 34-41.

20. Taylor RW, Gold E, Manning P \& Goulding A (1997) Gender differences in body fat content are present well before puberty. Int J Obes Relat Metab Disord 21, 1082-1084.

21. Dixon LB, Shannon BM, Tershakovec AM, Bennett MJ, Coates PM \& Cortner JA (1997) Effects of family history of heart disease, apolipoprotein E phenotype, and lipoprotein(a) on the response of children's plasma lipids to change in dietary lipids. Am J Clin Nutr 66, 1207-1217.

22. Kallio J, Pesonen U, Kaipio K, Karvonen MK, Jaakkola U, Heinonen OJ, Uusitupa MI \& Koulu M (2001) Altered 
intracellular processing and release of neuropeptide $\mathrm{Y}$ due to leucine 7 to proline 7 polymorphism in the signal peptide of preproneuropeptide $\mathrm{Y}$ in humans. FASEB J 15, 1242-1244.

23. Kallio J, Pesonen U, Karvonen MK, Kojima M, Hosoda H, Kangawa K \& Koulu M (2001) Enhanced exerciseinduced GH secretion in subjects with Pro7 substitution in the prepro-NPY. $J$ Clin Endocrinol Metab 86, 5348-5352.
24. Mattevi VS, Zembrzuski VM \& Hutz MH (2002) Association analysis of genes involved in the leptin-signaling pathway with obesity in Brazil. Int J Obes Relat Metab Disord 26, 1179-1185.

25. Porkka KVK, Viikari JSA, Rönnemaa T, Marniemi J \& Åkerblom HK (1994) Age and gender specific serum lipid and apolipoprotein fractiles of Finnish children and young adults. The Cardiovascular Risk in Young Finns Study. Acta Paediatr 83, 838-848. 